TTR

Traduction, terminologie, re?daction

\title{
Trois aspects de la combinatoire collocationnelle
}

\section{Jacqueline Bossé-Andrieu et Geneviève Mareschal}

Volume 11, numéro 1, 1er semestre 1998

Diachronie et synchronie

Diachronics and Synchronics

URI : https://id.erudit.org/iderudit/037320ar

DOI : https://doi.org/10.7202/037320ar

Aller au sommaire du numéro

\section{Éditeur(s)}

Association canadienne de traductologie

ISSN

0835-8443 (imprimé)

1708-2188 (numérique)

Découvrir la revue

Citer cet article

Bossé-Andrieu, J. \& Mareschal, G. (1998). Trois aspects de la combinatoire collocationnelle. TTR, 11(1), 157-171. https://doi.org/10.7202/037320ar

\section{Résumé de l'article}

Trois aspects de la combinatoire collocationnelle - Nous examinons ici trois aspects du lien qui unit les éléments des collocations, combinaisons qui se caractérisent par leur fréquence dans le discours et par leur prévisibilité pour le locuteur de langue maternelle. Nous montrons d'abord que la fréquence d'apparition simultanée de deux éléments dans le discours n'est pas propre aux collocations; la cooccurrence de deux éléments peut résulter de la réalité extralinguistique ou du fait qu'ils sont unis par un lien notionnel et référentiel. Dans le cas des collocations au sens strict, ce lien résulte d'une affinité consacrée par l'usage d'une communauté linguistique donnée. Nous montrons ensuite que, dans certaines collocations (ex. : en pleine croissance), il y a chevauchement - ou indissociabilité - des contraintes syntaxiques et des contraintes lexicales. Enfin, nous présentons plusieurs manifestations du caractère arbitraire - ou capricieux - du lien qui unit les éléments de certaines combinaisons : des quasi-synonymes n'appellent pas les mêmes collocatifs; sur le plan grammatical, on ne peut pas toujours passer d'une catégorie grammaticale à une autre (un sourire angélique, *sourire angéliquement); enfin des quasi-synonymes peuvent entrer dans certaines combinaisons synonymes (flambée/escalade des prix), mais non dans d'autres (les prix flambent/*les prix escaladent).
Tous droits réservés (C TTR: traduction, terminologie, rédaction — Les auteurs, 1998
Ce document est protégé par la loi sur le droit d'auteur. L’utilisation des services d'Érudit (y compris la reproduction) est assujettie à sa politique d'utilisation que vous pouvez consulter en ligne.

https://apropos.erudit.org/fr/usagers/politique-dutilisation/ 


\title{
Trois aspects de la combinatoire collocationnelle
}

\author{
Jacqueline Bossé-Andrieu \\ Geneviève Mareschal
}

Un des projets menés à l'École de traduction et d'interprétation de l'Université d'Ottawa est l'élaboration d'un dictionnaire des combinaisons du français courant, recueil appelé COMBIDIC. Dans ce dictionnaire, chaque entrée (mot-base) est divisée en deux parties : la première comprend les combinaisons syntaxiques du mot-base, c'est-àdire les combinaisons grammaticales possibles pour ce mot-base et propres à celui-ci (ex. : lutte contre [quelque chosed); la deuxième comporte les combinaisons lexicales usuelles - ou collocations - dans lesquelles entre le mot-base (ex. : mener une lutte). Le mot-base peut être un nom, un verbe ou un adjectif.

Les collocations sont généralement définies comme des combinaisons de lexèmes qui se retrouvent fréquemment dans le discours d'une communauté linguistique donnée'; ces lexèmes, qui s'attirent

\footnotetext{
'Exemples de collocations : pluie torrentielle, pleuvoir à verse, trempé jusqu'aux os. On peut noter que, a l'intérieur d'une communauté linguistique donnée, par exemple la communauté de langue française, il existe des variantes stylistiques et des variantes sémantiques. C'est ainsi que, an Canada, la collocation familière pleuvoir à boire debout existe à côté de ta collocation pleuvoir à verse, et que la collocation passer un examen a le plus souvent le sens de a réussir un examen ", alors que, pour un locuteur européen, cette collocation a le sens de * subir, se
} 
mutuellement sur les plans sémantique et stylistique, sont unis par un lien d'affinité plus ou moins contraignant. Ce lien permet de distinguer la " collocation au sens strict " (par exemple être muré dans son silence) de la " combinaison libre " (par exemple s'habituer au silence) et de la "locution figée "(par exemple passer sous silence).

Dans une * collocation au sens strict ", le lien entre les éléments, c'est-à-dire l'affinité entre les éléments ou la cohésion des éléments, est tel que le collocatif prend souvent - mais pas toujours ${ }^{2}-$ un sens qui résulte de sa combinaison avec le mot-base. Par exemple, dans la collocation esstyer un échec, le collocatif verbal essuyer prend le sens de " subir " en fonction du mot-base échec auquel il est uni. Toutefois, contrairement à la locution figée, les éléments d'une collocation gardent chacun leur autonomie sémantique et syntaxique. Ainsi, il est souvent possible de remplacer le collocatif par un synonyme (essuyer/subir un échec) et d'intercaler un ou plusieurs éléments entre le mot-base et le collocatif (essuyer un grave échec). Il faut noter que le lien entre les deux éléments d'une collocation est d'autant plus étroit et privilégié que la combinabilité du collocatif avec d'autres mots-bases est faible : le collocatif adverbial grièvement ne se combine qu'avec blessé et, comme le note Fakhour (1998), humeur massacrante est l'une des rares collocations dans lesquelles entre le collocatif adjectival massacrante (qui a le sens de " mauvaise "); dans ces deux cas, le collocatif attire autant le mot-base que le mot-base attire le collocatif, et la cohésion des éléments est très forte, et donc le lien très étroit ${ }^{3}$. Pour le locuteur dont la langue

présenter à un examen *.

${ }^{2}$ Dans la collocation grièvement blessé, le collocatif grièvement ne tire pas son sens de par sa combinaison avec blessé.

${ }^{3}$ Comme le constate Cruse (1986, p. 40), the constituent elements are, to varying degrees, mutually selective. The semantic integrity or cohesion of a collocation is the more marked if the meaning carried by one (or more) of its constituent elements is highly restricted contextually, and different from its meaning in more neutral contexts." Ainsi le verbe esstyyer, au sens de " subir ", se combine avec un nombre limité de noms (affront, échec, refus, tempête) qui expriment quelque chose de désagréable. 
maternelle est le français, la combinaison de humeur et de massacrante est prévisible en raison de sa récurrence dans le discours.

C'est le lien qui unit les éléments d'une collocation qui fait l'objet de la présente étude. Nous examinerons d'abord la nature de ce lien; puis nous montrerons qu'il est difficile, dans certains cas, de dissocier les contraintes syntaxiques liées au mot-base des contraintes syntaxiques liées au collocatif; nous montrerons finalement le caractère arbitraire ou capricieux du lien qui unit les éléments de certaines combinaisons.

\section{Nature du lien lexical qui unit les éléments des combinaisons}

Les collocations se caractérisent par la fréquence avec laquelle les deux éléments de la combinaison apparaissent ensemble dans le discours. Toutefois, la fréquence ne peut être le seul critère de sélection des collocations puisqu'elle peut résulter de liens de différente nature - linguistique ou extralinguistique - , mais pas nécessairement collocationnelle.

\section{1) Lien essentiellement extralinguistique et non collocationnel}

La fréquence d'apparition simultanée de deux lexèmes dans un corpus ${ }^{4}$ peut être due au fait que les rapports entre les éléments lexicaux sont dictés par la réalité extralinguistique. Les référents coexistent normalement, de par leur nature, dans la réalité, et le lien qui unit les deux éléments est essentiellement extralinguistique et non collocationnel. Ces combinaisons sont prévisibles du fait de la réalité dénotée par les mots et se rapprochent des combinaisons libres. Il existe différents exemples de combinaisons de ce type.

Ainsi, on relève avec une certaine fréquence des combinaisons du type Nom + de + Nom, telles que les lettres d'un mot, les syllabes d'un mot. Les collocatifs lettres, syllabes font partie de la même réalité que le nom-base mot puisque les lettres, les syllabes sont des parties d'un mot.

\footnotetext{
${ }^{4}$ Notre corpus comporte deux volets : un corpus dictionnairique (dictionnaires usuels) et un corpus textuel joumalistique.
} 
Les deux éléments de telles combinaisons sont liés par un lien métonymique (partie/tout), donc un lien essentiellement extralinguistique, et ne constituent pas des collocations. D'ailleurs, le fait que le mot-base peut être omis ou sous-entendu montre bien que ce ne sont pas des collocations (ex. : il a sauté une syllabe, une lettre). On peut parler des parties d'un tout sans mentionner ce tout. C'est le cas aussi de la paire $l e$ sens d'un mot, combinaison dictée par les propriétés du nom-base. Par opposition, la magie des mots est bel et bien une collocation puisque, dans cette combinaison, il y a une affinité linguistique entre les éléments. Le collocatif magie prend son sens particulier de * pouvoir/capacité de séduire " de par sa juxtaposition avec le mot-base; de plus, on ne peut omettre ce dernier.

De la même façon, les caractéristiques extralinguistiques d'un nom-base font que celui-ci entraîne sa combinaison avec certains verbes et en exclut d'autres. Ainsi, le nom porte apparait plusieurs fois dans le corpus à côté des verbes entrer et sortir. Mais, comme nous le verrons plus loin, ces combinaisons sont à la limite des collocations proprement dites. C'est essentiellement la réalité qui est à l'origine de la juxtaposition des éléments. Par opposition, une combinaison telle que proférer une menace est une véritable collocation, le choix de proférer, de préférence à dire, prononcer, est essentiellement linguistique et collocationnel. Il rêsulte d'une affinité dictée par l'usage d'une communauté linguistique donnée.

\section{2) Lien essentiellement linguistique et non collocationnel}

Dans certaines combinaisons du type Nom + de + Nom-base on Nombase $+\mathrm{de}+\mathrm{Nom}$, les deux éléments qui apparaissent ensemble dans le discours - et qui, graphiquement, constituent plusieurs éléments forment cependant, sur le plan sémantique, un tout indissociable parce qu'ils correspondent à une seule notion. Ils ne sont pas autonomes et l'ensemble constitue donc un terme et non une collocation. Prenons, pour illustrer cette opposition entre collocation et terme, les paires torrent de montagne et torrent de boue. La première n'est pas une collocation, mais une unité lexicale complexe, du type chemin de fer. Elle renvoie à une sorte de torrent, elle est indécomposable (*un torrent canadien de montagne). La seconde, dont le mot-base est boue, est une collocation. Elle est décomposable (un torrent imprévu de boue) et torrent prend le 
sens de * écoulement rapide et brutal * (Petit Robert) du fait de sa juxtaposition avec boue - tout comme il prend un sens autre que son sens premier avec des noms tels que eau, injures ou larmes, dans torrent d'eau, torrent d'injures ou torrent de larmes.

De même, dans certaines combinaisons du type Nom-base + Modificateur, les deux éléments constituent un terme. Par exemple, vis platinée est un terme et non une collocation. C'est une combinaison sémantiquement et syntaxiquement indécomposable, dont les deux éléments ne sont pas autonomes. Une vis platinée n'est pas à proprement parler une vis, mais la piêce d'un moteur qui, de nos jours, ne comporte plus de platine. Dans cheveux platinés, au contraire, nous avons deux éléments, et l'adjectif platiné prend son sens de "blond très pâle * au contact de cheveux. Autre exemple : sommeil paradoxal est un terme $\mathrm{e}^{5}$, alors que sommeil de plomb, sommeil profond, sommeil réparateur sont des collocations de la langue courante. D'ailleurs, le Nouveau Petit Robert donne le sens de sommeil paradoxal à l'entrée sommeil, ce qui prouve la non-autonomie des éléments et l'indécomposabilité de la combinaison; en revanche, le même dictionnaire donne la combinaison sommeil réparateur à l'entrée réparateur (sommeil, repos réparateur), ce qui prouve l'autonomie des éléments. Dans le cas des termes, le lien qui unit les lexèmes est essentiellement notionnel et référentiel.

3) Lien extralinguistique/linguistique et combinaisons à la limite de la collocation

Il convient de mentionner que, dans un grand nombre de cas, il est difficile de séparer le purement linguistique du purement extralinguistique. Il existe en effet une zone floue entre les combinaisons dictées par la réalité extralinguistique et les collocations dictées par un lien d'affinité linguistique. Ainsi, dans épeler, écrire, lire un mot, ou encore déclarer, gagner, perdre la guerre, les actions exprimées par le verbe sont liées naturellement, de par la réalité extralinguistique, au nombase. Certaines de ces combinaisons, résultant plus de la logique de la

s Sommeil paradoxal désigne, selon le Nouveau Petit Robert, " une phase du sommeil correspondant aux périodes de rêve, et où se produisent des mouvements oculaires très rapides *. 
réalité extralinguistique que d'une affinité consacrée par l'usage linguistique d'une communauté donnée, sont néanmoins des collocations. Même si le collocatif ne prend pas un sens autre que son sens premier au contact du mot-base, par opposition avec la collocation au sens strict du type essuyer un échec ou encore, exemple de Melc'uk (1993), donner une conférence, ce collocatif a une combinabilité restreinte - combinabilité dictée par la réalité extralinguistique. En effet, que peut-on faire avec des mots sinon les écrire, les épeler? Inversement, que peut-on écrire, épeler sinon des mots. En raison de la combinabilité restreinte de leurs éléments, les combinaisons du type écrire un mot, épeler un mot sont donc bel et bien des collocations. La même constatation vaut pour le nom-base porte et les collocatifs ouvrir ou fermer. Ces combinaisons sont, il est vrai, à la limite de la combinaison libre et de la collocation. Dictées par la réalité extralinguistique, elles partagent cependant avec la collocation les caractéristiques de combinabilité restreinte des constituants et de frequence dans le discours.

\section{IL. Liens syntaxiques}

Toutes les combinaisons lexicales obéissent à des règles syntaxiques. En effet, à toute collocation est attachée une structure qui obeit aux règles de la syntaxe française. C'est ainsi que les schémas syntaxiques suivants régissent les collocations :

. Nom (-base) + Modificateur (collocatif) (ex. : silence religiewx, de mort)

.Nom (-base) sujet + Verbe (collocatif) (ex. : le silence règne)

.Verbe (collocatif) + Nom (-base) c.o.d. (ex. : garder le silence)

.Verbe (-base) + Adverbe (collocatif) (ex. : se moquer éperdument)

.Adjectif (-base) + Adverbe (collocatif) (ex. : immensément riche)

.Nom + préposition + Nom (-base) (ex. : une lueur d'espoir)

En plus des contraintes syntaxiques ci-dessus propres à la langue, il y a, au niveau de la « micro-syntaxe ", c'est-à-dire à l'intérieur même d'une collocation donnée, des contraintes syntaxiques propres aux éléments qui la composent. Ces contraintes sont, d'une part, imposées par le mot-base; par exemple, dans mener la lutte contre quelqu'un/quelque chose, la 
préposition contre est imposée par le mot-base lutte. Ces contraintes sont, d'autre part, imposées par le collocatif; il s'agit encore ici essentiellement de prépositions; par exemple, dans renoncer à la lutte, la préposition à est imposée par le collocatif verbal renoncer. Cependant, dans certains cas, on se rend compte que la préposition qui précède le nom-base est dictée à la fois par le celui-ci et par le collocatif.

Si nous prenons l'exemple de guerre (au sens de " lutte, combat " mais non au sens de " lieu où se déroule la guerre n, de " champ de bataille $n$, de $\alpha$ front $"$ ), on constate que ce nom se construit avec certaines prépositions qui lui sont propres. Ainsi, les prépositions da avec, contre, de, entre lient le mot guerre aux mots qui suivent (ex. : guerre aux abus, avec un pays, d'indépendance, contre l'injustice, entre detax factions), tandis que la préposition en précède normalement ce nom (ex. : pays en guerre) pour indiquer un état ${ }^{6}$. Cependant, dans des collocations telles que entrer, partir en guerre (c'est-à-dire * en état de guerre "), la préposition en est imposée à la fois par le mot-base guerre et par le collocatif entrer ou partir puisque les verbes collocatifs entrer et partir - verbes qui désignent le passage d'un état à un autre - exigent aussi cette préposition. (Exemples : entrer en transe, partir en vacances.) Il y a donc ici indissociabilité des contraintes syntaxiques imposées par le mot-base de celles qu'impose le collocatif. Le même phénomène se manifeste dans certaines combinaisons comportant le nom-base navire : le nom navire se combine syntaxiquement avec la locution prépositive à bord de. Cette locution, interchangeable avec sur, est donc imposée par le mot-base navire ${ }^{7}$. Cependant, lorsque le mot-base navire se combine avec le collocatif verbal monter, qui impose lui aussi la locution prépositive $\grave{a}$ bord de ou la préposition sur, le résultat est la combinaison monter sur/ à bord d'un navire dans laquelle il y a indissociabilité de la construction

\footnotetext{
${ }^{6} \mathrm{Au}$ sens de * front *, le nom guerre est précédé de la préposition $a$ (ex : partir à la guerre, à la guerre comme à la guerre).

${ }^{7}$ La locution prépositive à bord de précède un moyen de locomotion (avion, navire, voiture). Avec navire, elle a le sens de * sur $\mathrm{m}$; avec avion, voiture, elle a le sens de * dans ".
} 
entraînée par le verbe et par le nom : les deux se partagent la même préposition. La préposition est dictée à la fois par le sémantisme du collocatif verbal et par celui du nom-base.

Signalons enfin un demier exemple, celui-ci avec le nom-base croissance. Un collocatif de croissance est l'adjectif pleine. Cependant, notre corpus montre que, contrairement à d'autres collocatifs adjectivaux comme forte, faible, rapide, l'adjectif pleine ne se combine avec croissance que si cette collocation est précédée de la préposition $e n$, préposition qui précède normalement le nom croissance. En effet, alors que l'on peut avoir, d'une part, des exemples tels que un marché, un secteur, des sociétés en croissance et, d'autre part, des exemples tels que la forte croissance, la croissance rapide (que connaît un secteur), notre corpus ne donne aucune occurrence de la pleine croissance (de quelque chose). Seule existe la double combinaison en + pleine + croissance. Dans en pleine croissance, il y a chevauchement - ou indissociabilité des contraintes syntaxiques imposées par le nom-base (en + croissance) et des contraintes imposées par la cooccurrence du collocatif adjectival pleine et du nom-base croissance.

\section{L'arbitraire - ou les caprices - de la combinabilité}

Le lien qui unit les éléments d'une combinaison est en grande partie arbitraire et capricieux. Prévisibles pour le locuteur natif en raison de leur récurrence dans le discours, les collocations d'une langue donnée sont par contre imprévisibles pour le locuteur étranger. $\mathrm{Si}$, pour un francophone, l'adjectif massacrante vient spontanément à l'esprit pour qualifier une " mauvaise " humeur, il n'en est pas de même pour un locuteur anglophone. Rien, pour ce dernier, ne permet de prévoir que, en français, c'est l'adjectif massacrante - et non " pourrie " (foul) comme en anglais - qui se combine avec $\propto$ humeur ${ }^{9}$. Le caractère arbitraire des

- Le verbe monter, qui indique un mouvement ascendant, se construit généralement avec la préposition sur (ex. : monter sur un trottoir, une chaise). La préposition sur est dictée par le sémantisme du verbe monter.

${ }^{2}$ Dans son article « Lexicologie contrastive et traduction ", Michel Paillard (1993, pp. 43-44) montre les difficultés posés par le * caractère plus ou moins exclusif * des collocations pour les traducteurs et les apprenants de l'anglais ou du français 
collocations apparaît donc de façon éclatante lorsqu'on compare les collocations de deux langues différentes et entraîne, dans des communautés bilingues, des calques tels que paver la voie, rencontrer des objectifs, collocations * transportées " de l'anglais au français d'autant plus facilement qu'il est difficile à un francophone de voir pourquoi ces combinaisons ne sont pas françaises. L'arbitraire - ou les caprices - de la combinatoire apparaît aussi lorsqu'on compare entre elles certaines des combinaisons du français courant ${ }^{10}$. Les divers exemples qui suivent illustrent cet arbitraire. Nous les avons divisés en trois catégories qui révèlent la manifestation de l'arbitraire : la première en fonction des propriétés lexico-sémantiques du mot-base, la deuxième en fonction des propriétés grammaticales du mot-base et la troisième en fonction de facteurs à la fois lexicaux et grammaticaux.

\section{1) Des quasi-synonymes n'appellent pas le même collocatif}

Sur le plan lexical, le lien qui unit un mot-base et ses collocatifs est généralement contraignant et exclusif. De sorte que des mots-bases synonymes ou quasi-synonymes ne se comportent pas de façon identique dans leur combinatoire collocationnelle. Ainsi, les collocatifs verbaux avec lesquels se combine un nom-base ne se combinent pas forcément avec un quasi-synonyme de ce nom. Par exemple, si le verbe déclarer se combine avec guerre (déclarer la guerre), il ne se combine toutefois pas avec lutte ("déclarer la lutte). Et on remarque aussi que l'affinité entre le verbe mener et le nom-base lutte semble plus grande (mener la lutte) que celle qui existe entre ce même verbe et guerre (mener la guerre). Un

langue étrangère. Et Mel'cuk (1993, pp. 100-101), dans " La phraséologie et son rôle dans l'enseignement/apprentissage d'une langue étrangère *, donne des exemples de combinaisons en langues étrangères dont il traduit littéralement les éléments en français : rain "pluie" + heavy "lourd" (anglais), Applause "applaudissements" + tosender "mugissant" (allemand), rajoda "espoir" + qabi "tenir" (somalien), etc. La traduction littérale de ces combinaisons donne des résultats surprenants pour le francophone.

${ }^{10} \mathrm{~L}$ 'arbitraire est encore plus flagrant dans le cas des locutions ou expressions dites figées. Comme le dit Rey (1987, X-XI) à propos de celles-ci, * le langage crée ses effets au moyen de choix subtils parmi des possibilités mille fois plus grandes $*$ 
même corpus textuel donne trois fois plus d'occurrences de la première collocation (mener la lutte) que de la seconde (mener la guerre).

Il ne nous paraît pas non plus sans intérêt de mentionner ici les variations que l'on relève, dans l'emploi de certaines collocations, dans deux communautés linguistiques qui partagent la même langue. Pour illustrer ce type de variations, prenons le cas de collocations dont les noms-bases sont discours et conférence. Les collocations données par les dictionnaires (Lexis et Petit Robert), aussi bien au mot-base qu'au collocatif, sont respectivement faire et prononcer pour le premier (faire, prononcer un discours) et faire et donner pour le second (faire, donner une conférence) - ce qui semble indiquer que le nom conférence n'a que peu d'affinité pour le collocatif prononcer, et discotrs peu d'affinité pour le collocatif donner. Un corpus français de France ne donne du reste que 2 occurrences de prononcer une conférence et 2 occurrences de donner un discours. En revanche, un corpus canadien donne 164 occurrences de prononcer une conférence et 31 de donner un discours. Il est vrai par ailleurs que la combinaison prononcer un discours est la plus fréquente dans les deux corpus ( 116 occurrences dans le corpus français de France et 529 dans le corpus canadien); de même, la combinaison donner une conférence est la plus courante ( 30 occurrences dans le corpus français de France et 371 dans le corpus canadien). On remarque donc que, même si les deux communautés linguistiques privilégient les mêmes collocations (prononcer un discours et donner une conférence), elles divergent cependant dans la fréquence d'emploi d'autres combinaisons comportant les mêmes mots-bases et des collocatifs synonymes ou quasi synonymes"

Le caractère arbitraire du lien qui unit les mots-bases et leurs collocatifs se manifeste aussi dans les combinaisons comportant un nom et un adjectif. En effet, deux noms quasi synonymes ne se combinent pas toujours avec le même collocatif adjectival. Par exemple, alors que le nom-base lutte se combine avec serrée (lutte serrée) et que guerre se combine avec larvée (guerre larvée), ni *lutte larvée ni *guerre serrée ne

"Ces données sont fournies par le corpus du Projet de Dictionnaire canadien bilingue, corpus français de 78,4 millions de mots, qui contient des textes de la Presse canadienne française et du Monde. 
sont attestées dans le corpus, pas plus d'ailleurs que *combat larvé ou *combat serré.

Dans tous les cas mentionnés ci-dessus, il semble difficile d'attribuer au seul sémantisme des mots-bases le rejet ou l'acceptation d'un collocatif par un synonyme ou un quasi-synonyme. Le plus souvent, on ne peut que constater l'arbitraire. On peut d'ailleurs noter qu'au niveau syntaxique aussi, un certain degré d'arbitraire existe puisque des quasisynonymes ne se construisent pas toujours de la même façon. Par exemple, selon les dictionnaires, guerre se construit avec les prépositions à ou contre (guerre à/contre le chômage), mais lutte, comme combat, ne se construit qu'avec la préposition contre. Sur le plan sémantique, il est difficile de justifier cette différence, ce qui explique en partie l'existence de la variante canadienne fréquente lutte au déficit, dans laquelle entre la préposition $\grave{a}$.

\section{2) Passage d'une catégorie à une autre}

Le caractère arbitraire et capricieux de la combinatoire collocationnelle se manifeste aussi sur le plan grammatical. Ainsi, on ne peut pas toujours passer d'une collocation à base nominale à une collocation à base verbale - ou vice-versa - en transférant le mot-base et le collocatif d'une catégorie à une autre. Par exemple, à côté des collocations à base nominale sourire béat et sourire diabolique, on trouve les collocations à base verbale sourire béatement et sourire diaboliquement; par contre, on ne trouve pas, à côté des collocations à base nominale large sourire et sourire angélique, les combinaisons à base verbale *sourire largement et *sourire angéliquement.

La même constatation vaut pour d'autres types de collocations. Pour donner un exemple du type Verbe (collocatif) + Nom (-base), citons deux exemples avec le nom-base guerre. Alors que l'on peut passer de déclarer la guerre a déclaration de guerre, on ne peut passer de la combinaison allumer la guerre - attestée dans notre corpus et appartenant selon Lexis à la langue littéraire - à la combinaison "allumage de la guerre. La métaphore qui existe dans la combinaison Verbe + Nom-base ne s'étend pas à la combinaison Nom + Nom-base. Cette différence est sans doute liée au fait que l'aire sémantique de allumer est plus grande que celle de allumage; tout comme l'aire sémantique de admettre est plus 
grande que celle de admission, ce qui fait que l'on peut dire les chiens ne sont pas admis dans le magasin (Lexis) ${ }^{12}$, mais pas ${ }^{*}$ 'admission des chiens est interdite dans le magasin. Dans le cas de allumer/allumage de la guerre, l'emploi métaphorique ne vaut que pour le verbe.

\section{3) Quasi-synonymes et passage d'une catégorie à une autre}

Un dernier exemple combinant à la fois un cas de quasi-synonymie et de passage d'une catégorie grammaticale à une autre montre encore le caractère arbitraire et capricieux du lien qui unit les éléments d'une collocation. Ainsi, on a bien flambée/escalade des prix, mais si on a les prix flambent, on n'a pas *les prix escaladent. Cette différence résulte de la construction exigée par les verbes et par le sémantisme du verbe et/ou du nom ; le verbe escalader est transitif (escalader une montagne), alors que le verbe flamber peut être transitif (flamber de l'argent) et intransitif (le bois flambe) et cet emploi intransitif permet les combinaisons dans lesquelles le nom fait l'action exprimée par le verbe ${ }^{13}$. Par ailleurs, on note que flamber peut avoir un sens figure, comme le substantif qui en est dérivé flambée (= montée soudaine). Par contre, escalader ne peut avoir, comme le nom escalade (dans escalade des prix) de sens figuré; le verbe escalader ne peut vouloir dire que " gravir, grimper sur * et est transitif; par conséquent, quelque chose - ici les prix - ne peut escalader. Escalade a deux sens, le sens propre (" escalade d'une montagne ") et le sens figuré ( " progression en violence ou en intensité, ascension "), mais escalader n'en a qu'un.

\footnotetext{
${ }^{12} \mathrm{Si}$, selon Lexis, « admettre " un être animé, c'est le laisser entrer dans un local, dans un lieu, dans un groupe ou une organisation, le nom * admission * ne s'applique qu'à l'entrée dans un groupe ou une organisation et non dans un local ou dans un lieu.
}

${ }^{13}$ Pour illustrer cette propriété, il suffit de donner l'exemple de verbes intransitifs tels que entrer et sortir (le roi entre $>$ l'entrée du roi; le roi arrive $>$ l'arrivée du roi). 


\section{Conclusion}

Au terme de cette exploration, on se rend compte de la complexité de la combinatoire collocationnelle. Nous avons montré qu'il est parfois difficile de dissocier la structure syntaxique propre au mot-base de celle qui est propre aux collocatifs de ce demier, et qu'il est souvent difficile de distinguer les liens entraînés par la réalité extralinguistique de ceux qui sont plus particulièrement dictés par une affinité linguistique, ce qui rend délicat le choix des collocations à inclure dans un ouvrage lexicographique ${ }^{i 4}$. Nous avons aussi montré l'aspect * arbitraire " ou capricieux de cette combinatoire même si ce qui semble être au premier abord des caprices peut parfois s'expliquer sur le plan sémantique et/ou grammatical. Comme le dit Fakhour (1998), qui étudie les groupes nominaux collocationnels français du type Nom + Adjectif en vue de leur traduction automatique, " les collocations ne constituent pas un phénomène simple à identifier mais un ensemble d'éléments hétéroclites $\%$.

Université d'Ottawa

\section{Références}

CRUSE, D.A. (1986). Lexical Semantics. Cambridge Texbooks in Linguistics, Cambridge, University Press.

FAKHOUR, Sabah (1998). * Definition et traduction automatique des collocations N-Adj ». Tanger, Turjuman, 7,1, pp. 59-70.

Le Nouveau Petit Robert (1993). Paris, Dictionnaires Le Robert.

LEXIS. Larousse de la langue française (1979). Paris, Librairie Larousse.

MEL'CUK, Igor (1993). « La phraséologie et son rôle dans

\footnotetext{
${ }^{14}$ Ces difficultés se posent aussi dans l'élaboration des entrées du Dictionnaire canadien bilingue, projet dirigé par R.P. Roberts, à l'Université d'Ottawa. Dans ce cas, il s'agit de distinguer les combinaisons qui doivent être placées parmi les combinaisons libres, de celles qui doivent figurer comme collocations et de celles qui doivent figurer comme composés.
} 
l'enseignement/apprentissage d'une langue étrangère \%. Études de linguistique appliquée, 92, pp. 82-113.

PAILLARD, Michel (1993). " Lexicologie contrastive et traduction ". La traduction à l'Université. Recherches et propositions didactiques. Michel Ballard éd. Lille, Presses de l'Université de Lille, Collection UL.3, pp. 3146.

REY, Alain et Sophie CHANTREAU (1987). Dictionnaire des expressions et locutions. Paris. Dictionnaires Le Robert.

RÉSUMÉ : Trois aspects de la combinatoire collocationnelle - Nous examinons ici trois aspects du lien qui unit les éléments des collocations, combinaisons qui se caractérisent par leur fréquence dans le discours et par leur prévisibilité pour le locuteur de langue maternelle. Nous montrons d'abord que la fréquence d'apparition simultanée de deux éléments dans le discours n'est pas propre aux collocations; la cooccurrence de deux éléments peut résulter de la réalité extralinguistique ou du fait qu'ils sont unis par un lien notionnel et référentiel. Dans le cas des collocations au sens strict, ce lien résulte d'une affinité consacrée par l'usage d'une communauté linguistique donnée. Nous montrons ensuite que, dans certaines collocations (ex. : en pleine croissance), il y a chevauchement - ou indissociabilité - des contraintes syntaxiques et des contraintes lexicales. Enfin, nous présentons plusieurs manifestations du caractère arbitraire - ou capricieux - du lien qui unit les éléments de certaines combinaisons : des quasi-synonymes n'appellent pas les mêmes collocatifs; sur le plan grammatical, on ne peut pas toujours passer d'une catégorie grammaticale à une autre (un sourire angélique, ${ }^{*}$ sourire angéliquement); enfin des quasi-synonymes peuvent entrer dans certaines combinaisons synonymes (fambée/escalade des prix), mais non dans d'autres (les prix flambent/"les prix escaladent).

ABSTRACT : Three Aspects of Collocations Combinations - We examine three aspects of the link which binds together the constituents of collocations. By collocations, we mean combinations of two lexical items which appear together frequently in texts and which are therefore predictable for a native speaker. We first show that cooccurence is a characteristic shared with other associations, such as terms whose elements cooccur because of an extralinguistic reality; in these 
associations, the link is purely notional and referential and does not result from the usage of a given linguistic community. Then we show that in some collocations, such as en pleine croissance, syntactic and lexical constraints cannot be dissociated. Finally we present several cases showing the arbitrary character of the link which binds collocations and makes them unpredictable for the non-native speaker : words which are near-synonyms do not combine with the same collocates; it is not always possible to switch from a collocation with a given grammatical base to another (un sourire angélique, *sourire angéliquement); finally words which are near-synonyms can be combined in collocations of two nouns (flambée/escalade des prix) but not in combinations of noun plus verb (les prix flambent/*les prix escaladent).

Jacqueline Bossé-Andrieu et Geneviève Mareschal : École de traduction et d'interprétation, Université d'Ottawa, Ottawa, Ontario, KIN 6N5. Courriel : jbosse@uottawa.ca 